\title{
Testing quantum effects of gravity and dark energy at laboratory scales
}

\section{Sivaram $^{1}$, Kenath Arun ${ }^{* 2}$, Avijeet Prasad ${ }^{3}$}

${ }^{1}$ Indian Institute of Astrophysics, Bangalore, 560 034, India, e-mail: sivaram@iiap.res.in ${ }^{2}$ CHRIST (Deemed to be University), Bangalore, 560 029, India, e-mail: kenath.arun@cjc.christcollege.edu

${ }^{3}$ Center for Space Plasma \& Aeronomic Research, The University of Alabama in Huntsville, Huntsville, Alabama 35899, USA, email: ap0162@uah.edu

\begin{abstract}
One of the biggest challenges in modern physics is how to unify gravity with quantum theory. There is an absence of a complete quantum theory of gravity, and conventionally it is thought that the effects of quantum gravity occur only at high energies (Planck scale). Here we suggest that certain novel quantum effects of gravity can become significant even at lower energies and could be tested at laboratory scales. We also suggest a few indirect effects of dark energy that can show up at laboratory scales. Using these ideas, we set observational constraints on radio recombination lines of the Rydberg atoms. We further suggest that high-precision measurements of Casimir effects for smaller plate separation could also show some manifestations of the presence of dark energy.
\end{abstract}

Keywords: Quantum gravity; dark energy; Rydberg atoms; Casimir effect

\footnotetext{
${ }^{*}$ Corresponding author
} 


\section{Introduction}

One of the most challenging open questions in modern physics is to describe gravity through quantum mechanics $[1,2]$. The current understanding of gravity is based on the general theory of relativity (in the framework of classical physics). However, this description is incomplete as quantum mechanics is considered to be more fundamental. Although there are several different approaches to the problem of quantizing gravity, no fully consistent theory is yet to emerge [3]. Even in the absence of such a complete theory, there are interesting implications of quantum gravity that are testable. So far, the proposed tests of quantum effects of gravity have focussed on specific models, phenomenology, and cosmological observations [4-7].

Normally it is thought that quantum effects of gravity will show up only at the Planck energy (of $\sim 10^{19} \mathrm{GeV}$ ). However, Planck energies (or scales) are likely to remain inaccessible in the foreseeable future. To accelerate particles to Planck scale, the energies required are very high. Using the most intense lasers of intensity $\sim 10^{26} \mathrm{~W} / \mathrm{m}^{2}$, the arm of the linear accelerator will have to be a few light-years to achieve Planck energies. Even in cosmic rays we do not see such high energy particles (maximum energy being $\sim 10^{21} \mathrm{eV}$ ) [8]. So we are left looking for testability at lower energies, and on laboratory scales.

There have been numerous experimental confirmations of Einstein's theory of relativity from observations of massive astronomical objects and their dynamics, such as the direct detection of gravitational waves from the merger of two black holes and neutron stars $[9,10]$. Also, laboratory experiments such as the tests of the equivalence principle, precision measurements of gravitational constant, validity of Newton's law at micro-scales, have been continuously increasing. A recent experiment [11] shows the gravitational coupling between two gold spheres of 1 millimetre radius, which extends the gravity measurements to small, single source masses and to low gravitational field strengths. This provides a viable path to explore a regime of gravitational physics that involves precision tests of gravity of microscopic masses at around the Planck mass $\left(\sim 10^{-5} g\right)$. This could help us in understanding how gravity fits with quantum mechanics on smaller scales.

Here, we consider some new quantum effects of gravity and their testability at laboratory scales, without going to Planck energies (scales). We also discuss in this context, the possibility of looking for effects of dark energy (cosmological constant due to quantum fluctuation) at atomic (laboratory) scales. 


\section{Quantum effects and modification of Newtonian gravity}

In a possible unified description of gravity and quantum theory, since quantum theory is more general (with classical theory being a special case), the role of the uncertainty principle should be fundamental [12]. As we go to smaller scales, the momentum increases and a wave packet of wavelength $\lambda$ will have an effective mass given by $h / \lambda c$. Further, a particle of mass $m$ cannot be localized to a distance less than $h / m c$, which is the spread of the wave packet.

Now, if two quantum particles come closer and closer till they are separated by a distance $r$, then the uncertainty principle implies that their mutual gravitational force becomes, $F=G \frac{\left(\frac{\hbar}{r c}\right)\left(\frac{\hbar}{r c}\right)}{r^{2}}=\frac{\hbar^{2} G}{c^{2}} \frac{1}{r^{4}}=\frac{\hbar c}{r^{4}}\left(\frac{\hbar G}{c^{3}}\right)$. This gives:

$F=\frac{\hbar c L_{p l}^{2}}{r^{4}}$

where, $L_{p l}=\sqrt{\frac{\hbar G}{c^{3}}}$ is the Planck length.

So, we have a $\frac{1}{r^{4}}$ dependence rather than the usual Newtonian $\frac{1}{r^{2}}$. Therefore, at short distances, the gravitational force would be very different from the classical case. Testing with smaller and smaller masses on smaller scales could shed some light on such quantum modifications of gravity. This force will be maximum at the Planck length. Hence when $r=L_{p l}$ in equation (1), we have the maximum force,

$F_{\text {max }}=\frac{c^{4}}{G}$

To get an estimate of the magnitude of this force, we note that at the beta decay length of $r_{\beta}=10^{-17} \mathrm{~cm}, F_{\beta} \approx 8 \times 10^{-15}$ dyne and at a scale of the proton Compton wavelength of $\sim 2 \times 10^{-14} \mathrm{~cm}$, the corresponding force will be $\approx 5 \times 10^{-28} d y n e$. For the current experimental detection limit of force, $F_{\text {limit }} \approx 10^{-19}$ dyne [13], the corresponding length scale will be, $r_{\text {limit }} \approx 2 \times 10^{-16} \mathrm{~cm}$.

This modification may also have consequences for avoiding the singularity in black hole collapse. Since the particles can't come closer due to the uncertainty principle, they can't be localized to smaller distances. Indeed, it turns out this maximal force given by equation (2) would imply a finite radius (for the collapsing mass inside the horizon) of,

$r_{\text {min }}=\left(\frac{G M}{a_{\max }}\right)^{1 / 2}$

where $a_{\max }$ is the maximum acceleration (field strength) corresponding to maximum force $F_{\max }[14-16]$. 


\section{Dark energy can limit the size and energies of Rydberg atoms}

Can the effects of dark energy manifest in limiting sizes of Rydberg atoms? We have the general relativistic Reissner-Nordström solution for a particle of mass $m$ and charge $e$. When the cosmological constant $\Lambda$ (considered to be dark energy) is included in the energymomentum tensor, we still have an exact solution (sometimes referred to as the Kottler metric). This solution has a $g_{00}$ component given as:

$g_{00}=1-\frac{2 G m}{r c^{2}}+\frac{G e^{2}}{c^{4} r^{2}}-\frac{\Lambda r^{2}}{3}$

For electron mass, $m=m_{e}$, the second term is negligible. If $e$ is the electron charge, the third and fourth terms, i.e. the electrostatic and dark energy terms become comparable for a region with size $r$, give as:

$\frac{G e^{2}}{c^{4} r^{2}}=\frac{\Lambda r^{2}}{3}$

$r^{4}=\frac{3 G e^{2}}{\Lambda c^{4}}$

or $r=\left(\frac{3 G e^{2}}{\Lambda c^{4}}\right)^{1 / 4} \approx 10^{-3} \mathrm{~cm}$.

Physically this would imply that for an electron, the two terms become comparable for a region of this extent. Now Rydberg atoms (those atoms with high principal quantum number, $n$ ) can have sizes of this order [17]. The atomic radius of these atoms is:

$r=\frac{n^{2} \hbar^{2}}{m_{e} e^{2}} \approx n^{2} r_{B}$

where $r_{B}$ is the Bohr radius. Combining equations (6) and (7), we get:

$r \approx 10^{-3} \mathrm{~cm}, n<10^{3}$

These Rydberg atomic states are well observed in astrophysics as radio recombination lines, since the transition energy involved are in the radio wavelengths. So far, the highest $n$ observed is around 700, which is consistent with equations (7) and (8) [18]. In other words, the fact that dark energy density and electrostatic energy density become comparable for atoms of this size, could be a possible reason why we do not observe higher $n$ hydrogen recombination lines.

We can also consider heavier atoms, i.e. with higher atomic number $Z$. In this case the nuclear charge of these atoms will be $Z e$. With a charge of $Z e$, the balance between electrostatic and dark energy would occur at a value of $r$ given by:

$r=\left(\frac{3 G Z e^{2}}{\Lambda c^{4}}\right)^{1 / 4}$ 
For $Z=12$, this would give about twice the radius given by equation (6), which gives $r \approx$ $1.8 \times 10^{-3} \mathrm{~cm}$. But since the size of the higher $Z$ Rydberg atoms would be:

$r=\frac{n^{2} \hbar^{2}}{m_{e} Z e^{2}}$.

In this case the limiting $n$ - when dark energy density is comparable to electrostatic energy density - would have a dependence on the atomic number given as,

$n \propto Z^{5 / 8}$.

This then leads to a higher limit on $n$ as compared to that for the hydrogen recombination lines.

For $Z=12$, this limit on $n$ would be $<(12)^{5 / 8} \times 10^{3} \approx 4.7 \times 10^{3}$, which is consistent with the highest observed carbon recombination lines [19]. This balance of forces could be tested with experiments with single ions or electrons in devices like Penning traps etc. There could thus be manifestations of dark energy at laboratory scales.

Casimir effects when tested over sub-micron scales could reveal anomalies or deviations from expected results due to quantum vacuum background. In Casimir effect, the force between two plates becomes significant, with the force per unit area given as:

$\frac{F_{C a s}}{A}=\frac{\pi^{2} \hbar c}{240 r^{4}}$.

This is a purely quantum effect independent of any coupling. With the background dark energy density, the force (given by equation (12)) becomes important at a separation of $\sim 10^{-4}-$ $10^{-5} \mathrm{~cm}$, which is one-two orders less than that obtained from equation (8). These effects can come under the purview of future high precision measurements of Casimir effect, and hence the Casimir effect, when tested for smaller plate separation, could show some manifestation of the presence of the dark energy background.

\section{Conclusion}

Here, we have considered some new quantum effects of gravity and how they can be tested without having to achieve Planck energies. In this context, we have also shown the possibility of looking for effects of dark energy also at atomic scales. We point out the possible tests for the quantum effects of gravity at laboratory scales including the manifestations of dark energy. This could well have consequences for atomic physics, especially for large $n$ Rydberg atom. We also set limits on the radio recombination lines of such atoms, which are consistent with observations. We further predict that the limit of highest $n$ for higher $Z$ atoms will be higher, scaling as $Z^{5} / 8$. Finally, we mention that the future high-precision measurements of the Casimir effect could also show some manifestations of dark energy, which are again testable. 


\section{References:}

1. B. Schulz, arXiv:1409.7977v1 [gr-qc], 2014

2. R. Penrose, Gen. Rel. Grav., 28, 581, 1996

3. C. Kiefer, Annalen Phys., 15, 129, 2005

4. S. Hossenfelder, arXiv:1010.3420v1 [gr-qc], 2010

5. A. Ashoorioon, P. S. B. Dev and A. Mazumdar, Mod. Phys. Lett. A, 29, 1450163, 2014

6. I. Pikovski et al, Nature Physics, 8, 393, 2012

7. A. Albrecht, A. Retzker and M. B. Plenio, Phys. Rev. A, 90, 033834, 2014

8. J. Linsley, Phys. Rev. Lett., 10, 146, 1963

9. B. P. Abbott et al., Phys. Rev. Lett., 116, 061102, 2016

10. B. P. Abbott et al., Astrophys. J., 848, L12, 2017

11. T. Westphal et al., Nature, 591, 225, 2021

12. V. de Sabbata, C. Sivaram, H. H. Borzeszkowski and H. J. Treder, Annalen Phys., 503, 497, 1991

13. M. J. Biercuk, arXiv:1004.0780v2 [quant-ph], 2010

14. V. de Sabbata and C. Sivaram, Found. Phys. Lett., 6, 561, 1993

15. C. Sivaram and K. Arun, Astrophys. Space Sci., 337, 169, 2012

16. C. Sivaram, Astrophys. Space Sci., 167, 335, 1990

17. C. Sivaram, arXiv:1607.08114v1 [gr-qc], 2016

18. F. B. Dunning et al., J. Phys. B, 42, 022001, 2009

19. S. V. Stepkin et al., Mon. Not. R. Astron. Soc., 374, 852, 2007 